\title{
Galerkin finite element methods for wave problems
}

\author{
T K SENGUPTA $^{1}$, S B TALLA ${ }^{1}$ and S C PRADHAN ${ }^{2}$ \\ ${ }^{1}$ Department of Aerospace Engineering, Indian Institute of Technology, \\ Kanpur 208 016, India \\ ${ }^{2}$ Institute of High Performance Computing, 1, Science Park Road, \\ Singapore 117528 \\ e-mail: tksen@iitk.ac.in
}

MS received 31 September 2004; revised 3 March 2005

\begin{abstract}
We compare here the accuracy, stability and wave propagation properties of a few Galerkin methods. The basic Galerkin methods with piecewise linear basis functions (called G1FEM here) and quadratic basis functions (called G2FEM) have been compared with the streamwise-upwind Petrov Galerkin (SUPG) method for their ability to solve wave problems. It is shown here that when the piecewise linear basis functions are replaced by quadratic polynomials, the stencils become much larger (involving five overlapping elements), with only a very small increase in spectral accuracy. It is also shown that all the three Galerkin methods have restricted ranges of wave numbers and circular frequencies over which the numerical dispersion relation matches with the physical dispersion relation - a central requirement for wave problems. The model one-dimensional convection equation is solved with a very fine uniform grid to show the above properties. With the help of discontinuous initial condition, we also investigate the Gibbs' phenomenon for these methods.
\end{abstract}

Keywords. $\quad h p$-Finite element method; continuous Galerkin methods; wave solutions; Gibbs' phenomenon.

\section{Introduction}

Galerkin methods belong to the class of solution methods for PDEs where the solution residue is minimized giving rise to the well-known weak formulation of problems. In this approach, the dependent variable, $u(x, t)$, for a one-dimensional space-time dependent problem is expressed in an expansion of the form,

$$
u(x, t)=\sum_{l=1}^{N} \phi_{l}(x) u_{l}(t),
$$

where $\phi_{1}, \ldots \phi_{N}$ are the predetermined expansion or basis functions. Unlike spectral methods, where $\phi_{j}$ 's are the chosen global orthogonal functions, in finite element methods, basis functions are taken as low-order polynomials that are localized about the chosen elements. 
These methods, developed in engineering, have now been used in many diverse applications including meteorology, oceanography and many other scientific disciplines that require tracking various wave phenomena. In the present work, we investigate the usefulness of the Galerkin FEM methods for their suitability in solving wave problems.

In the Galerkin approach, the problem is reformulated by requiring the equation residual to be orthogonal to each of the basis functions that are chosen from a Lagrange family of interpolation functions. The basis functions satisfy the natural boundary conditions.

Considerable amount of research has been undertaken in recent times to solve hyperbolic problems to develop optimal methods with respect to local mesh size $h$ and the local polynomial degree $p$. The resulting methods have, hence, been termed $h p$-finite element methods. The comparison between continuous piecewise ploynomials (CGFEM) and their discontinuous versions can be found in Houston et al (2002), where a least-squares stabilization method is proposed for discontinuous Galerkin FEM methods. One of the major problems with CGFEM is its poor numerical stability that shows up as nonphysical oscillations. This has been attempted to be removed by the streamline-upwind/ Petrov-Galerkin (SUPG) formulation of Brookes \& Hughes (1982) that implicitly incorporates numerical dissipation in the characteristic direction. However, it has been shown by Sengupta (2004) that the introduction of low-order dissipation terms, as in SUPG, can significantly alters the solution and this can be a significant deterrent in using a stabilized methods like this for solving wave problems.

If we are interested in assessing the quality of results for wave problems, then it is natural to solve the one-dimensional wave equation given by,

$$
(\partial u / \partial t)+c(\partial u / \partial x)=0, \quad c>0
$$

that represents a wave moving from left to right at a phase speed, $c$. The particular benefit of choosing the above equation as a model equation rests on the fact that it admits an exact solution that shows the initial condition as convecting at the phase speed. Thus, if we choose a discontinuous initial condition, then the numerical solution of the above equation demonstrates the method's ability to solve the wave problem correctly. This property of the solution for the non-dissipative wave propagation of above helps in callibrating different numerical methods, a procedure followed by Sengupta (2004). Rigorous error analysis of SUPG and its $h p$-version for first-order hyperbolic problems is reported by Johnson et al (1984) and Houston et al (2002) respectively. Error bounds have been reported for hyperbolic problems that are simultaneously optimal with respect to the local mesh size and the local polynomial degree. In the present work, instead of providing error bounds etc., we propose to characterize a few Galerkin methods by comparing the exact solution with the numerically obtained solutions using a uniform fine mesh, with special emphasis on wave problems and the Gibbs' phenomenon.

For space-time dependent problems, where convection dominates, as for Navier-Stokes equation, the dispersion relation preservation (DRP) property is of utmost importance. In Sengupta et al (2003) and Sengupta (2004) this property has been defined in terms of group velocity. Also, Sengupta et al (2003) introduce a new global spectral analysis tool by which different numerical methods can be analysed. The same methodology is used here for analysing the Galerkin methods. For the analysis of (2), one can represent the dependent variable in terms of its Fourier-Laplace transform by,

$$
u(x, t)=\int U(k, \omega) e^{i(k x-\omega t)} \mathrm{d} k \mathrm{~d} \omega .
$$


Substitution of this in (2) yields the physical dispersion relation, $\omega=k c$ that relates the spatial and temporal scales. However, in discrete computations we have an equivalent numerical dispersion given by,

$$
\omega=k_{e q} c_{N}
$$

In the next section, we provide an estimate for $k_{e q}$ and $c_{N}$ for Galerkin FEM methods that use piecewise linear and quadratic basis functions for solving the one-dimensional wave equation.

\section{One-dimensional wave equation}

First, we briefly discuss the Galerkin method that employs piecewise quadratic polynomials for the basis or interpolating functions. We will call this as G2FEM for ease of reference. Here, one would have three quadratic functions for each element (see Reddy 2001, for details). In figure 1, we show the basis functions for the five elements that are needed to discretize Equation (2). The basis functions have non-zero components defined by,

$$
\begin{aligned}
(x+h)(x+3 h / 2) /\left(h^{2} / 2\right), & \text { for } \quad(l-3 / 2) h \leq x \leq(l-1 / 2) h, \\
\phi_{l}(x)=-4(x+h / 2)(x-h / 2) / h^{2}, & \text { for } \quad(l-1 / 2) h \leq x \leq(l+1 / 2) h, \\
& (x-h)(x-3 h / 2) / h^{2} / 2, \quad \text { for } \quad(l+1 / 2) h \leq x \leq(l+3 / 2) h .
\end{aligned}
$$

The Galerkin method involves substituting (5) in (2) and requiring the equation residue to be orthogonal to the basis function $\phi_{l}(x)$, to obtain the discrete equation. This yields the following discrete equation for the interior nodes (for $3 \leq l \leq N-2$ ) as

$$
\begin{aligned}
{\left[-\frac{\mathrm{d} u_{l+2}}{\mathrm{~d} t}+4 \frac{\mathrm{d} u_{l+1}}{\mathrm{~d} t}+24 \frac{\mathrm{d} u_{l}}{\mathrm{~d} t}\right.} & \left.+4 \frac{\mathrm{d} u_{l-1}}{\mathrm{~d} t}-\frac{\mathrm{d} u_{l-2}}{\mathrm{~d} t}\right] \\
& +\frac{5 c}{2 h}\left(u_{l-2}-8 u_{l-1}+8 u_{l+1}-u_{l+2}\right)=0 .
\end{aligned}
$$

We can contrast this stencil with the one for the Galerkin method that uses piecewise linear interpolation functions (henceforth called G1FEM) that is given here for the interior nodes (for $2 \leq l \leq N-1$ ) by,

$$
\left[\frac{\mathrm{d} u_{l+1}}{\mathrm{~d} t}+4 \frac{\mathrm{d} u_{l}}{\mathrm{~d} t}+\frac{\mathrm{d} u_{l-1}}{\mathrm{~d} t}\right]+\frac{3 c}{h}\left(u_{l+1}-u_{l-1}\right)=0 .
$$

Note that the spatial derivative parts of (6) and (7) correspond to fourth-order and secondorder accurate stencils, that we obtain using finite difference methods. However, the temporal derivatives, as appearing in (6) and (7) make the discrete equation more accurate than what the spatial discretization may seem to reveals. This issue of enhanced spectral accuracy has been discussed earlier by Grescho \& Sani (1998) for FEM exclusively, and recently by Sengupta (2004) for FEM in comparison to other discrete computing methods.

For the SUPG method, the discrete equation, for $2 \leq l \leq N-1$, is quoted here from Grescho \& Sani (1998) as,

$$
\begin{aligned}
(1+ & \beta / 2) \frac{\mathrm{d} u_{l-1}}{\mathrm{~d} t}+4 \frac{\mathrm{d} u_{l}}{\mathrm{~d} t}+(1-\beta / 2) \frac{\mathrm{d} u_{l+1}}{\mathrm{~d} t}+\frac{3 c}{h}\left(u_{l+1}-u_{l-1}\right) \\
& =\frac{6 \beta c}{h^{2}}\left(u_{l+1}-2 u_{l}+u_{l-1}\right),
\end{aligned}
$$



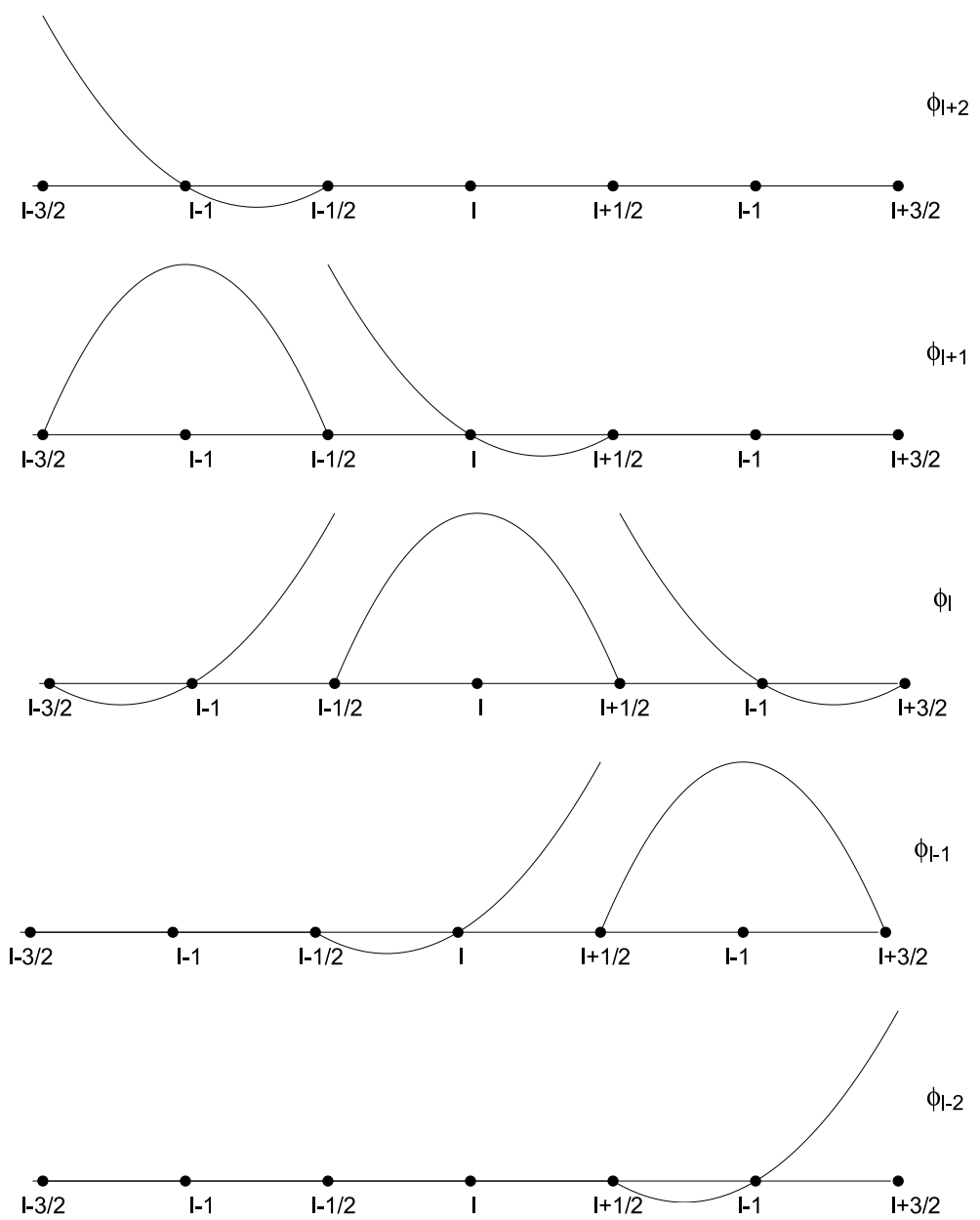

Figure 1. One-dimensional Lagrange quadratic elements and their interpolation functions. Shown are the participating five elements for discretizing differential equation for the $i$ th node.

where $\beta$ is the streamwise-diffusion parameter, that will be used here as $0 \cdot 26$, based on the work of Raymond \& Garder (1976), that suggested that this value of the parameter corresponds to least phase error. The right hand side of (8) represents the lowest order dissipation term. If one chooses $\beta=1 / 2$, then one recovers the discrete equation for first-order upwind finite difference scheme.

To evaluate $k_{e q}$ of the discrete equations (6)-(8), we redefine

$$
u(x, t)=\int \hat{U}(k, t) e^{i k x} \mathrm{~d} k
$$

Substitution of (9) in (2) yields,

$$
\begin{aligned}
& \frac{\mathrm{d} \hat{U}}{\mathrm{~d} t}\left(-e^{2 i k h}+4 e^{i k h}+24+4 e^{-i k h}-e^{-2 i k h}\right) \\
& \quad+\frac{5 c}{2 h}\left(e^{-2 i k h}-8 e^{-i k h}+8 e^{i k h}-e^{2 i k h}\right) \hat{U}=0 .
\end{aligned}
$$


Thus, the equivalent wave number for G2FEM is given by,

$$
k_{e q}^{(2)}(l)=\frac{5(4-\cos k h) \sin k h}{h(12+4 \cos k h-\cos 2 k h)} .
$$

We can similarly obtain the corresponding $k_{e q}$ for G1FEM and SUPG respectively (as given by Sengupta 2004) by

$$
k_{e q}^{(1)}(l)=(3 \sin k h) /[h(2+\cos k h)],
$$

and

$$
k_{e q}^{S U P G}(l)=(6 / h)[\sin k h-i \beta(1-\cos k h)] /[(4+2 \cos k h-i \beta \sin k h)] .
$$

The physical significance of $k_{e q}$ lies in its utility in revealing the spectral accuracy of the numerical method. The essential ideas are given in Sengupta (2004), while the specific cases of Galerkin FEM method are provided here. It is customary to plot $k_{e q} / k$ against $k h$ up to the Nyquist limit $(k h=\pi)$. To achieve spectral accuracy, this quotient should ideally be equal to one, and any departure from this constitutes phase error. All the estimates provided above are for the interior nodes, because the discrete equations are for the interior points only. For the boundary and near-boundary points $(l=1$ and 2), we need to alter the basis functions, such that the interpolation properties are satisfied. In figure 2 , one such possible alteration is used for the basis functions $\phi_{1}$ and $\phi_{2}$ are shown. The basis functions are given for $l=1$ as,

$$
\phi_{1}=(3 h-2 x) / h, \quad \text { for } h \leq x \leq 3 h / 2,
$$

and

$$
=(x-2 h)(2 x-5 h) / h^{2}, \quad \text { for } 3 h / 2 \leq x \leq 5 h / 2 .
$$

similarly, the basis functions for $l=2$ are given by,

$$
\begin{aligned}
\phi_{2} & =2(x-h) / h, \quad \text { for } h \leq x \leq 3 h / 2, \\
& =(2 x-3 h)(5 h-2 x) / h^{2}, \quad \text { for } 3 h / 2 \leq x \leq 5 h / 2,
\end{aligned}
$$

and

$$
=(x-3 h)(2 x-7 h) / h^{2}, \quad \text { for } 5 h / 2 \leq x \leq 7 h / 2 .
$$

When we use these basis functions, the discrete equations for (2) is obtained as,

$$
\left[18 \frac{\mathrm{d} u_{1}}{\mathrm{~d} t}+9 \frac{\mathrm{d} u_{2}}{\mathrm{~d} t}-2 \frac{\mathrm{d} u_{3}}{\mathrm{~d} t}\right]+\frac{5 c}{h}\left(-6 u_{1}+7 u_{2}-u_{3}\right)=0
$$

for $l=1$ and

$$
\left[9 \frac{d u_{1}}{d t}+50 \frac{d u_{2}}{d t}+8 \frac{d u_{3}}{d t}-2 \frac{d u_{4}}{d t}\right]+\frac{5 c}{h}\left(-7 u_{1}+8 u_{3}-u_{4}\right)=0,
$$

for the $l=2$ element. In similar fashion we can obtain the discrete equations for $l=N-1$ and $N$ elements, that are not reported here. 

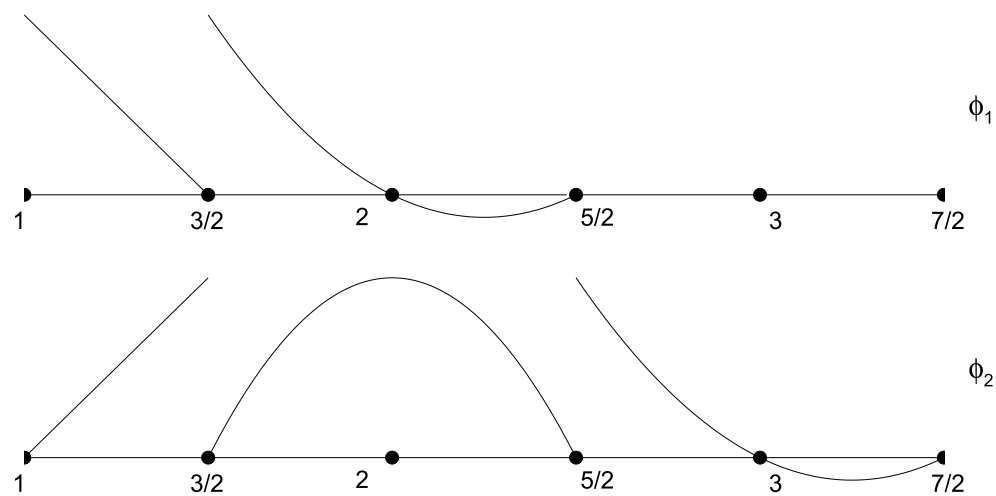

Figure 2. One-dimensional Lagrange quadratic elements and interpolation functions. Shown are the boundary and near-boundary elements.

\section{Results and discussion}

First and foremost, we would investigate the spectral accuracy provided by G1FEM, G2FEM and SUPG, by plotting $k_{e q} / k$ for the wave number range of $k h$ equal to 0 to $\pi$, as shown in figure 3. It is seen that the expressions given in (11) and (12) are purely real for the interior stencils. For the SUPG method, this is a complex quantity for all the nodes, as the associated stencil given by (8) is asymmetric. The imaginary part of $k_{e q} / k$ signifies added numerical dissipation, when this is negative for the wave equation (2). In contrast, a positive imaginary part would add anti-diffusion and hence would destabilize computations. Even when the Galerkin method is employed to discretize PDEs for boundary or near-boundary points, we notice that the discretized stencils are asymmetric. Hence, these also would indicate addition of implicit numerical dissipation or anti-diffusion, as the evaluated quantity's sign would indicate. The interesting aspect of the present analysis tool (that was developed and reported by Sengupta (2004) and Sengupta et al 2003) is that it provides information about the accuracy and numerical stability on a scale-by-scale basis. Thus, this method of analysis is a significant improvement over the classical von Neumann stability analysis.

In figure $3 a$ the spectral accuracy of G2FEM is compared with G1FEM and SUPG, by plotting the real part of $k_{e q} / k$ against $k h$. For SUPG and G1FEM methods only the accuracy of the interior stencil has been shown, while for the G2FEM the effect of boundary closure is also displayed along with the information for the interior stencil. It is seen that the interior stencil of G2FEM method displays lesser phase error as compared to the other two methods. Because of the alteration of the stencils for the boundary and near-boundary points, there is a lower spectral accuracy for these two points. It is to be noted that if it is required to incorporate Dirichlet boundary conditions, then we can dispense with the discrete equation (14) for the boundary stencil and not be affected by the poor spectral accuracy of this stencil. Also note that the addition of the streamwise upwind factor in (8) provides a negligible increase in spectral accuracy, as compared to G1FEM. This factor was chosen by Raymond \& Garder (1976) to enhance the spectral accuracy. However, we see that this term degrades the DRP property and quality of solution for the wave problem. There is a marginal increase of spectral accuracy, when one uses G2FEM instead of G1FEM.

In figure $3 \mathrm{~b}$, the imaginary parts of $k_{e q} / k$ are plotted for G2FEM and SUPG method. As mentioned before, G2FEM displays numerical dissipation (or anti-diffusion) only for the 

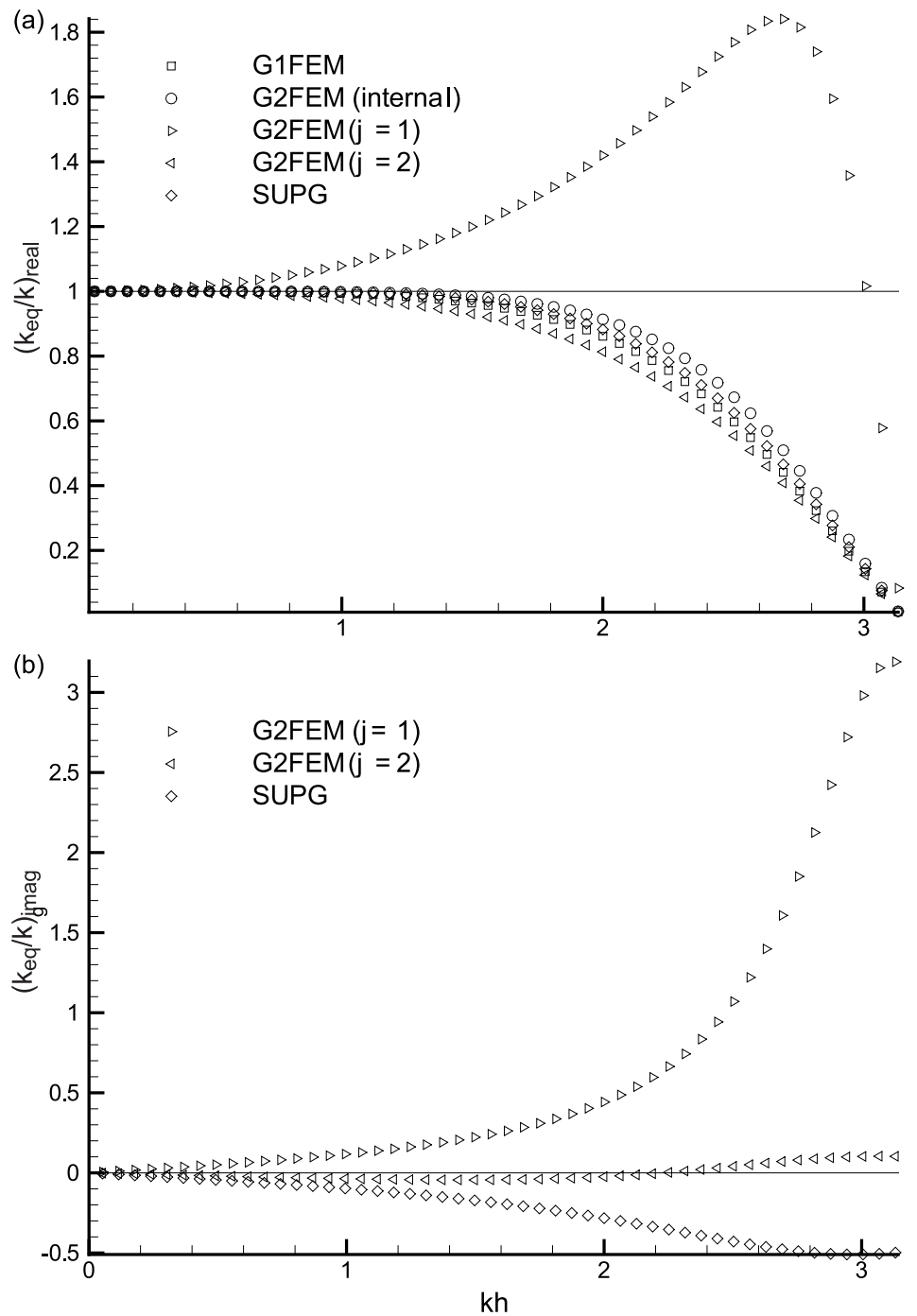

Figure 3. Spectral accuracy of FEM schemes. Shown are (a) the real part indicating phase error and (b) the imaginary part indicating added numerical dissipation.

boundary and near-boundary points, while SUPG method has in-built numerical dissipation for all points. For the governing equation (2), the signal propagates from left to right, while the used basis functions, as shown in figure 2, are one-sided with the left side of the function deficient with respect to the right side about the centre of the element. Such a choice of basis functions forces additional weightage of the signal on the right side, implying information propagation from right to left for these boundary and near-boundary elements. This opposite bias of the basis functions near the boundary leads to numerical instability. This is clearly evident from figure $3 \mathrm{~b}$ for the $l=1$ node, where all wave numbers add anti-diffusion. In contrast, for the second node, the plotted quantity in figure $3 \mathrm{~b}$, shows the method to be unstable only for high wave numbers, where it is positive. At lower wave numbers, the added small 
quantity of dissipation would stabilize large wavelengths. The amount of dissipation added in SUPG is significantly large as compared to the above dissipation at $l=2$. While this added dissipation would reduce numerical error, it would also attenuate the signal. Ideally, for any computations, we would therefore look for a neutrally stable algorithm for numerically solving wave problems. The amplification factors of the investigated schemes follow.

For space-time dependent problems, apart from spectral accuracy, we would also be interested in preserving the physical dispersion relation in a numerical sense for wave propagation problems. This aspect can be investigated in terms of the numerical phase speed and group velocity. by

For the wave equation (2), any chosen numerical scheme has an amplification factor given

$$
G=|G| e^{-i \gamma}
$$

so that the unknown at any arbitrary time can be written as,

$$
u\left(x_{m}, t^{n}\right)=\int u_{0}(k)[|G|]^{n} e^{i\left(k x_{m}-n \gamma\right)} \mathrm{d} k,
$$

where $u_{0}(k)$ is the Fourier transform of the initial condition. Thus the numerical phase speed is given by $c_{N}=\omega_{N} / k=\gamma / k \Delta t$, while the numerical dispersion relation is given by $\omega_{N}=k c_{N}$. Thus, the numerical group velocity is obtained as,

$$
V_{g N}=\mathrm{d} \omega_{N} / \mathrm{d} k=c_{N}+k\left(\mathrm{~d} c_{N} / \mathrm{d} k\right)
$$

Thus, the non-dimensional group velocity is obtained as,

$$
\frac{V_{g N}}{c}=\frac{c_{N}}{c}+\frac{k^{2}}{\omega} \frac{\mathrm{d}}{\mathrm{d} k}\left(\frac{\gamma}{k \Delta t}\right) .
$$

As,

$$
\frac{k^{2}}{\omega} \frac{\mathrm{d}}{\mathrm{d} k}\left(\frac{\gamma}{k \Delta t}\right)=\frac{k}{\omega \Delta t} \frac{\mathrm{d} \gamma}{\mathrm{d} k}-\frac{c_{N}}{c},
$$

so

$$
\frac{V_{g N}}{c}=\frac{k}{\omega \Delta t} \frac{\mathrm{d} \gamma}{\mathrm{d} k}=\frac{N_{c}^{-1}}{h} \frac{\mathrm{d} \gamma}{\mathrm{d} k},
$$

where $N_{c}$ is the CFL number. For G2FEM, at the interior stencils, we can show that the amplification factor of the method is given by

$$
G=1-\frac{5 i N_{c}(4-\cos k h) \sin k h}{(12+4 \cos k h-\cos 2 k h)} .
$$

Hence, the phase of this amplification factor is given by,

$$
\gamma_{2}=\tan ^{-1} \frac{5 N_{c}(4-\cos k h) \sin k h}{(12+4 \cos k h-\cos 2 k h)} .
$$

For G1FEM, the amplification factor for the interior stencil is given by (see Sengupta 2004, for details),

$$
G=1-\left[3 i N_{c} \sin k h /(2+\cos k h)\right]
$$


so that

$$
\gamma_{1}=\tan ^{-1}\left[3 N_{c} \sin k h /(2+\cos k h)\right] .
$$

Thus, using (20) and (22) in (18), one can obtain the non-dimensional numerical group velocity. Ideally, this should be equal to one and any departure from this indicates an improper propagation speed for energy. In figure 4, some selective contour-lines have been plotted for
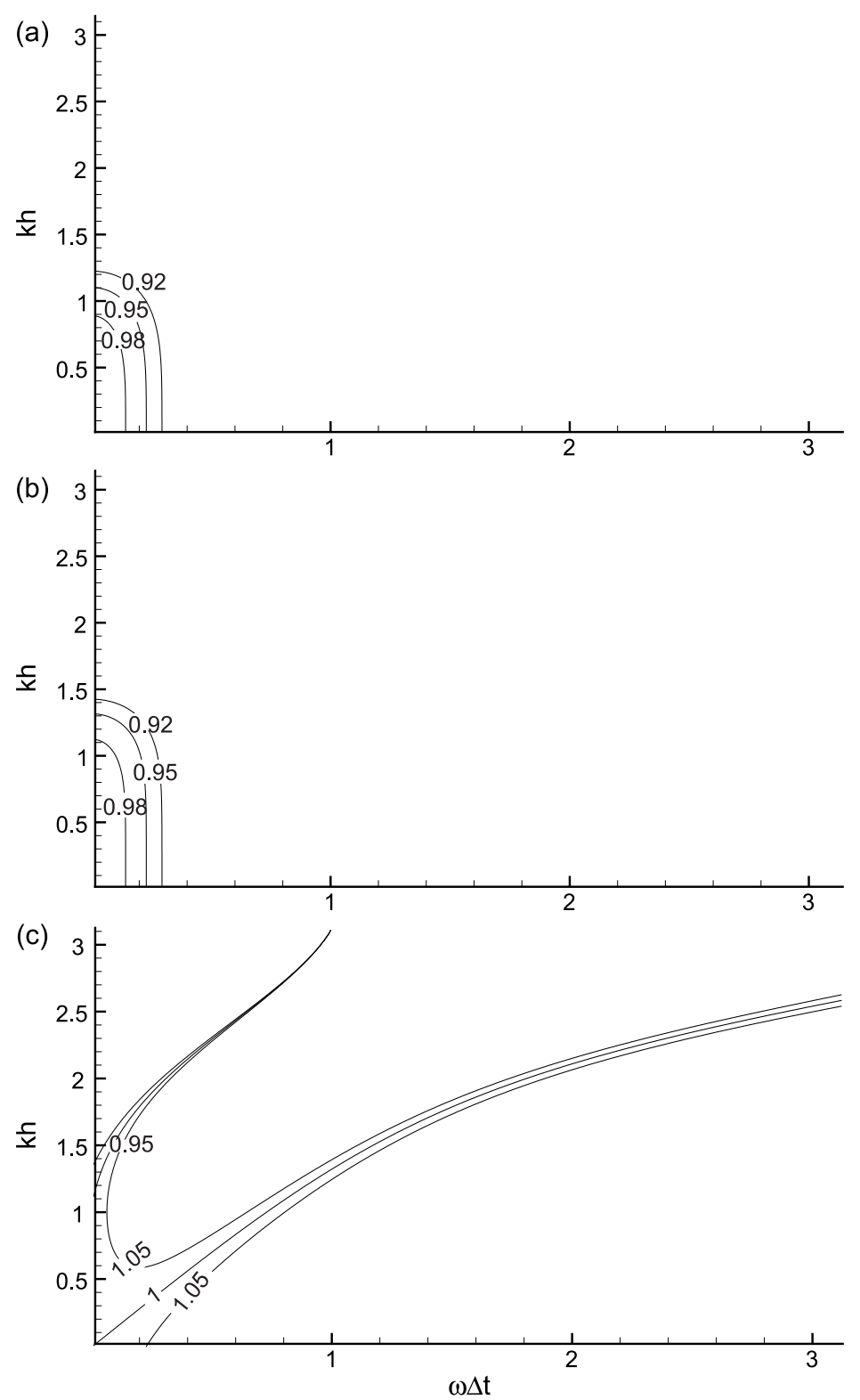

Figure 4. DRP property for Galerkin methods: (a) with linear interpolation functions, (b) with quadratic interpolation functions and (c) SUPG method in the $(k h-\omega \Delta t)$-plane for explicit Euler time-integration scheme. 

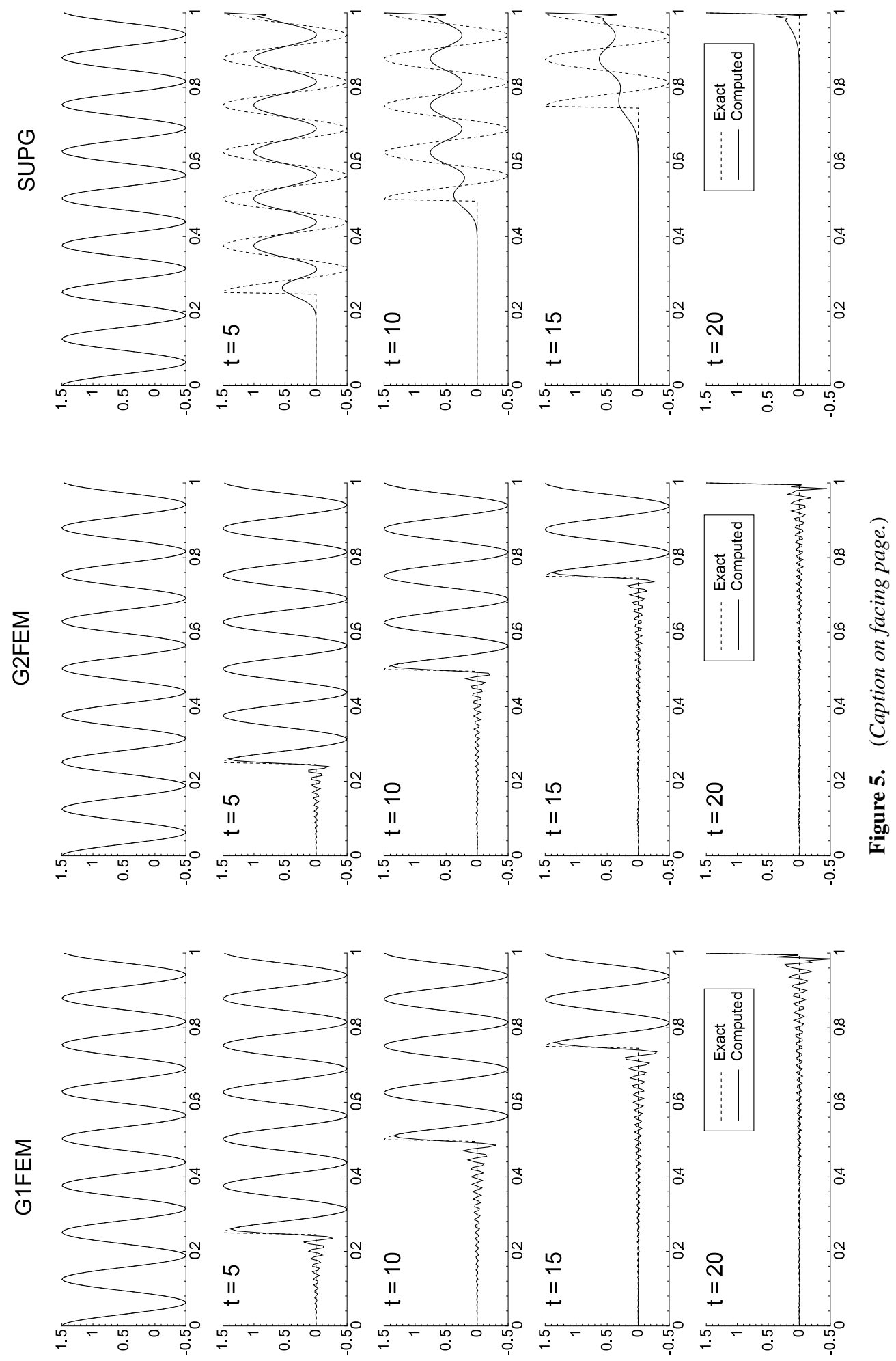


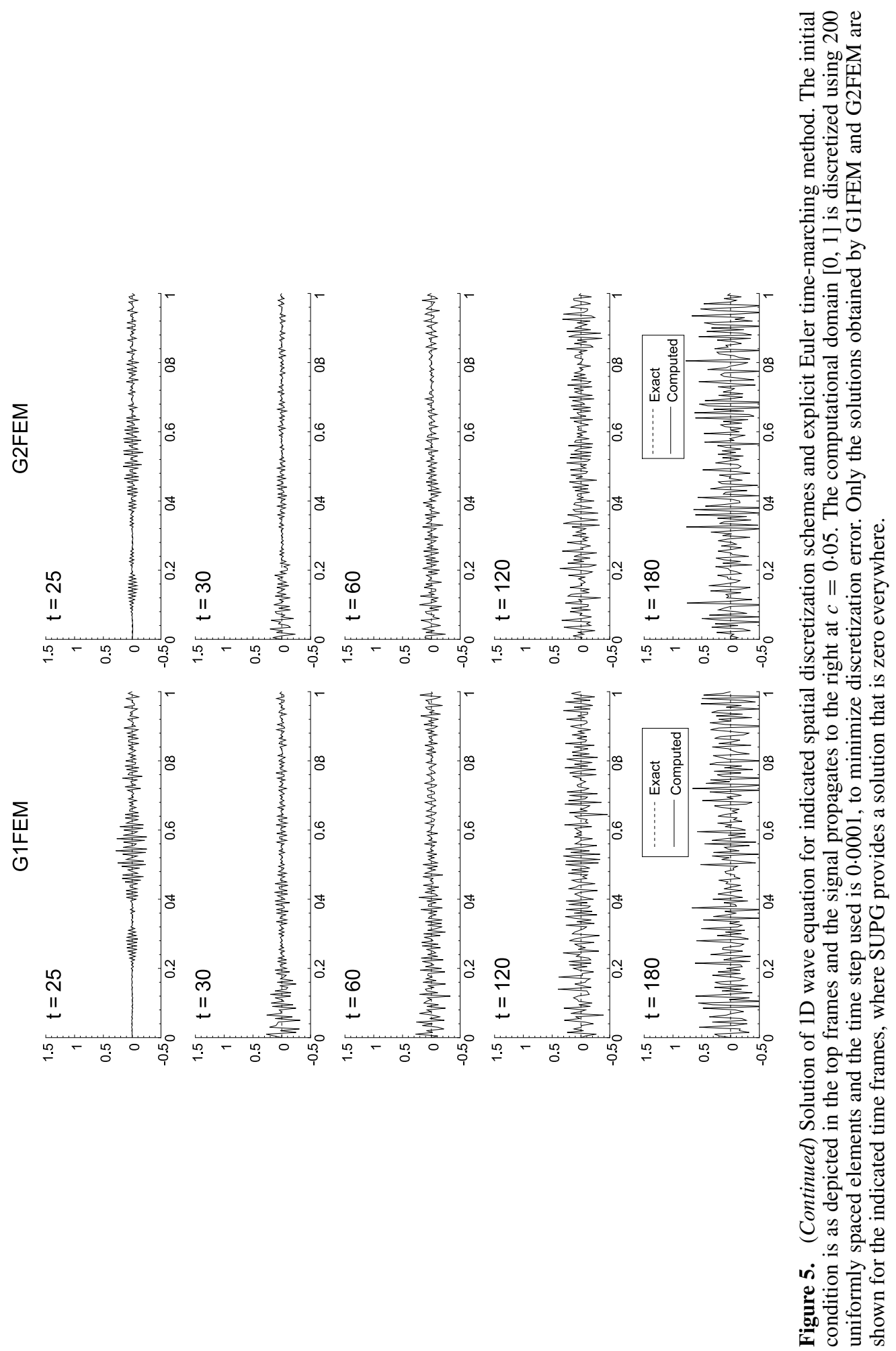


$V_{g N} / c$ for G1FEM, SUPG and G2FEM. It is clear from figure 4 that these methods have limited ranges of wave numbers over which the methods have acceptable DRP property. The range of circular frequencies over which DRP property is acceptable is also limited for the used Euler time integration scheme. Also, the range over which these schemes retain their DRP property without any solution distortion, is determined by the contiguous area near the origin in the $(k h-\omega \Delta t)$ - plane. Hence, of the three methods shown here, SUPG supports the DRP property over the smallest range of wave numbers and circular frequencies.

Finally, we would like to solve (2) by these three Galerkin methods and discuss their relative merits and demerits. In figure 5 the solution of the wave equation is displayed in a domain $[0,1]$ where we have used 201 equi-spaced points. The initial conditions used are displayed in the top frame and have jump discontinuities at both ends. The solution outside the domain is taken as zero and as the wave propagates from left to right, the boundary conditions have been taken as the Dirichlet conditions obtained from the exact solution. In this process, we avoid using the stencil (14), that we have seen from figure $3 \mathrm{~b}$ as unstable. For G2FEM, the stencils used are given by (15) and (6). The stencils for G1FEM and SUPG are given by (7) and (8) respectively. In these figures, the exact solution is shown by dotted lines in all the frames. The displayed solution indicates two sources of error. One is due to the added numerical dissipation that is clearly visible in the SUPG solution. The massive quantity of dissipation added by the right hand side term of (8) drastically attenuates the signal and with time the problem becomes worse. In contrast, G1FEM and G2FEM solutions are not dissipative as the interior stencils are symmetric and involve no numerical dissipation. However, these two methods suffer numerical instability from the near-boundary stencils. This is accentuated by the Gibbs' phenomenon that would appear while trying to compute a discontinuous solution with a nondissipative method. This phenomenon is observed in the vicinity of the discontinuity and shows up as non-physical oscillations. The magnitude and direction of propagation of this oscillation is determined by the truncation error of the method. An estimate of this for G1FEM is given in Sengupta (2004) and it is shown that the discontinuity is a source of oscillation that travels from right to left. Here, one can clearly see this train of oscillations from the discontinuity that travels upstream. Once it reaches the left boundary, it starts magnifying upon a reflection. This growing oscillation keeps increasing with time and for G1FEM, the displayed solution at $t=120$ and beyond shows this all pervading instability. As we have seen that G2FEM has larger spectral accuracy and hence it creates Gibbs' phenomena of smaller magnitude. This is evident in all the earlier time frames, where the Gibbs' phenomenon is seen to be slightly lower for G2FEM in comparison with G1FEM. However, once the whole domain is covered by unstable waves beyond $t=30$, no distinction between G1FEM and G2FEM can be made. This is due to the fact that G2FEM has marginal improvement of spectral accuracy and hence phase error over G1FEM, as shown in figure 3a. Note that the SUPG method, with added dissipation everywhere, suppresses the Gibbs' phenomenon completely. But the excessive dissipation, apart from controlling instability also attenuates the signal. As the solution is dissipated to zero within the computational domain beyond $t=20$, it is not shown for the SUPG method in figure 5.

\section{Conclusion}

In this note, we have compared the accuracy, stability and wave propagation properties of a few Galerkin methods. Basic Galerkin methods are non-dissipative and can be related to various finite difference methods when spatial discretization is compared. The Galerkin method with 
piecewise linear basis functions (called G1FEM here) only involves two neighbouring cells. But the overall method provides spectral accuracy that is of the same quality that is obtained from a tenth-order accurate explicit finite difference scheme. This is possible due to the temporal discretization process used in FEM. In a sense, this is akin to compact schemes used for high accuracy finite difference methods (see ch. 11 of Sengupta (2004) for details on compact schemes). However, in the Galerkin method, when the piecewise linear basis functions are replaced by quadratic polynomials, the stencils become larger (involving five overlapping elements), with only a very small increase in spectral accuracy, as shown in figure 3a. The latter method has been called G2FEM here.

There is also a specific problem with FEM discretization for wave problems near the boundaries. The basis functions are by necessity one-sided near the boundary and this makes the stencils asymmetric with anti-diffusion near one of the boundaries. This makes the pure Galerkin methods unusable for wave problems. It would appear that this can be solved by implicitly adding numerical dissipation everywhere, a procedure similar to that used in the SUPG method. Unfortunately, this will not be useful because the added massive lowest order dissipation will also attenuate the signal. For the simple case of the one-dimensional wave equation, this is shown in figure 5, where the loss of information is drastic, making the method practically useless. The initial condition chosen for this problem has discontinuity at the domain boundaries and computing this involves Gibbs' phenomenon. For G1FEM and G2FEM, this phenomenon accentuates the numerical instability problem, that is also discussed here.

\section{References}

Brooks A N, Hughes T J R 1982 Streamline upwind/Petrov-Galerkin formulations for convection dominated flows with particular emphasis on the incompressible Navier-Stokes equations. Comput. Meth. Appl. Mech. Engrg. 32: 199-259

Grescho P M, Sani R L 1998 Incompressible flow and the finite element method (Chichester: Wiley)

Houston P, Jensen M, Süli E 2002 hp-Discontinuous Galerkin finite element methods with least squares stabilization. J. Sci. Comput. 17 (1-4): 3-25

Johnson C, Nävert U, Pitkäranta J 1984 Finite element methods for linear hyperbolic problems. Comput. Meth. Appl. Mech. Engrg. 45: 285-312

Raymond W H, Garder A 1976 Selective damping in a Galerkin method for solving wave problems with variable grids. Mon. Weather Rev. 104: 1583-1590

Reddy J N 2001 An introduction to the finite element method (New York: McGraw-Hill)

Sengupta T K 2004 Foundations of computational fluid dynamics (Hyderabad: Universities Press)

Sengupta T K, Ganerwal G, De S 2003 Analysis of central and compact schemes. J. Comput. Phys. 192: $677-694$ 